\title{
Polymersomes eradicating intracellular bacteria
}

Federico Fenaroli ${ }^{1, \#}$, James D. Robertson ${ }^{2,3,4, \#}$, Edoardo Scarpa ${ }^{2,5}$, Virginia M. Gouveia ${ }^{2,5}$, Claudia Di Guglielmo 6 , Cesare De Pace ${ }^{2,5}$, Philip M. Elks ${ }^{3,4}$, Alessandro Poma ${ }^{2}$, Dimitrios Evangelopoulos $^{7}$, Julio Ortiz Canseco ${ }^{7}$, Helen M. Marriott ${ }^{4,7}$, David H. Dockrell ${ }^{4,7}$, Simon J. Foster $^{7,8}$, Timothy McHugh ${ }^{7}$, Stephen A. Renshaw ${ }^{3,4,1,8,10}$ Josep Samitier Martí ${ }^{6,11,12}$, Giuseppe Battaglia $^{2,5,6,13,{ }^{*}}$ and Loris Rizzello ${ }^{2,6,14^{*}}$

${ }^{1}$ Department of Biosciences, University of Oslo, Blindernveien 31, 0371 Oslo, Norway

${ }^{2}$ Department of Chemistry, University College London, WC1H OAJ - London (UK).

${ }^{3}$ Department of Biomedical Science, University of Sheffield, S10 2TN - Sheffield (UK).

${ }^{4}$ Department of Infection, Immunity, and Cardiovascular Disease, University of Sheffield Medical School, S10 2JF - Sheffield (UK).

${ }^{5}$ Institute for Physics of Living System, University College London, WC1E 6BT - London (UK).

${ }^{6}$ Institute for Bioengineering of Catalonia (IBEC), The Barcelona Institute of Science and Technology, Barcelona (Spain).

${ }^{7}$ Department of Clinical Microbiology, University College London, Royal Free Hospital, NW3 2PF

- London (UK).

${ }^{8}$ The Florey Institute, University of Sheffield, S10 2TN - Sheffield (UK).

${ }^{9}$ Department of Molecular Biology and Biotechnology, University of Sheffield, S10 2TN - Sheffield (UK).

${ }^{10}$ The Bateson Centre, University of Sheffield, Firth Court - S10 2TN - Sheffield (UK).

${ }^{11}$ Department of Electronics and Biomedical Engineering, University of Barcelona, Barcelona, Spain

${ }^{12}$ Networking Biomedical Research Center for Bioengineering, Biomaterials and Nanomedicine (CIBER-BBN), Madrid, Spain

${ }^{13}$ Catalan Institution for Research and Advanced Studies (ICREA), Barcelona, Spain.

${ }^{14}$ Department of Pharmaceutical Sciences, University of Milan, via Mangiagalli 25, 20133, Milano (Italy)

\# These authors equally contributed to the work

* Corresponding authors:

- Prof. Giuseppe Battaglia

- Dr. Loris Rizzello

E-mail:

g.battaglia@ucl.ac.uk and gbattaglia@ibecbarcelona.eu

Irizzello@ibecbarcelona.eu
Tel: $+44(0) 2076794688$

Tel: $+44(0) 7459381835$ 


\begin{abstract}
Mononuclear phagocytes such as monocytes, tissue-specific macrophages and dendritic cells are primary actors in both innate and adaptive immunity. These professional phagocytes can be parasitized by intracellular bacteria, turning them from housekeepers to hiding places and favoring chronic and/or disseminated infection. One of the most infamous is the bacteria that cause tuberculosis (TB), which is the most pandemic and one of the deadliest diseases with one third of the world's population infected, and an average of 1.8 million deaths/year worldwide.

Here we demonstrate the effective targeting and intracellular delivery of antibiotics to infected macrophages both in vitro and in vivo, using $\mathrm{pH}$ sensitive nanoscopic polymersomes made of PMPC-PDPA block copolymer. Polymersomes showed the ability to significantly enhance the efficacy of the antibiotics killing Mycobacterium bovis, Mycobacterium tuberculosis and another established intracellular pathogen the Staphylococcus aureus. Moreover, they demonstrated to easily access TB-like granuloma tissues - one of the harshest environments to penetrate - in zebrafish models. We thus successfully exploited this targeting for the effective eradication of several intracellular bacteria, including the M. tuberculosis - the etiological agent of human TB.
\end{abstract}

\title{
Keywords
}

Polymersomes; intracellular pathogens; tuberculosis; zebrafish; drug delivery

The human innate immune system - our frontline defense against potential pathogens - includes a range of effector cells. ${ }^{1}$ Examples are professional phagocytes, such as granulocytes (i.e. basophils, eosinophils and neutrophils) and mononuclear phagocytes (macrophages, dendritic cells, and monocytes). Phagocytes are responsible for the clearance of bacterial pathogens from the host and have attracted much interest in the context of focused antimicrobial drug delivery. In parallel, some of the deadliest pathogens have acquired the ability to evade the phagocytes' unique panel of molecular defenses. While phagocytes have evolved to eradicate invading pathogens, few selected bacteria have evolved strategies to make macrophages as their preferential niche evading host killing. Such a strategy is known as the 'macrophage paradox' and it is the product of millions of years of co-evolution. ${ }^{2,3}$ Pathogens may inhabit different compartments in the macrophage. Listeria monocytogenes, Shigella flexneri and the Rickettsiae rickettsii proliferate within the macrophage cytosol, ${ }^{4}$ Listeria pneumophila colonizes the ER-like vacuoles, ${ }^{5}$ and Salmonella enterica exploits the late endosomal compartments. ${ }^{6}$ More recently, a similar strategy has been reported for Staphylococcus aureus, suggesting that these bacteria are capable of hiding within professional phagocytes. ${ }^{7,8}$ The most studied intracellular pathogen Mycobacterium tuberculosis, survives within macrophage phagosomes otherwise a detrimental environment for most pathogens. ${ }^{9,10}$ Yet, M. tuberculosis has evolved creating proteins that hinder phagosome maturation preventing its fusion with lysosomes. ${ }^{9,11}$ The first-line therapy against TB includes an antibiotic combination regimen of isoniazid, rifampicin, pyrazinamide, and ethambutol for long time (six to nine months). Such duration of the therapies promoted a dramatic rise in multidrug-resistant (MDR)-TB, despite the recent approval of the bedaquiline as new approach for MDR-TB treatment. ${ }^{12,13}$ Also, antibacterial drugs have been demonstrated to accumulate within specific compartments of host cells, like the bedaquiline which is stored in host lipid droplets. ${ }^{14}$ These seems to act as an antibiotic reservoir that could be transferred to bacteria during host lipid consumption. ${ }^{15,16}$ It is evident that a way to improve drug efficacy is to encapsulate the active agent into a carrier that delivers it into the infected cell. Also, the optimal drug delivery systems should incorporate targeting specificity for 
the host cells type and should be able to cross biological barriers with the aim to finally reach the intracellular niche where the microorganisms hide - even more critical today with the emergence of drug resistant strains.

We propose here the use of synthetic vesicles, known as polymersomes, ${ }^{17}$ that can target infected phagocytes, to reach intracellular pathogens in their sub-cellular compartment, and to locally release their antibacterial cargo. These polymersomes are formed through the self-assembly of amphiphilic copolymers in aqueous media and combine the advantages of long-term stability with the potential to encapsulate a broad range compounds (or cargos). ${ }^{18-22}$ The $\mathrm{pH}$-sensitive block copolymer poly(2-(methacryloyloxy) ethyl-phosphorylcholine)-co-poly(2(diisopropylamino)ethyl methacrylate) (PMPC-PDPA) can combine specific cellular targeting in non-professional phagocytic cells (through the PMPC affinity toward the scavenger receptor B1) ${ }^{23}$ with effective endosomal and cytosolic drug delivery following internalization (by the $\mathrm{pH}$ sensitive PDPA). ${ }^{21,24-27}$

In this work, we explored the dynamics of the cellular uptake of PMPC-PDPA polymersomes in professional phagocytes, and their intracellular trafficking. As in vivo model for polymersomes distribution and accumulation, we chose the transparent zebrafish embryo infected with Mycobacterium marinum. ${ }^{28}$ In this system, the availability of specific cell lines allows real-time imaging of nanoparticles with the cell of interest such as leucocytes. Here, we described the ability of polymersomes to efficiently target macrophages in vivo, and to co-localize with their intracellular pathogens. We made further experiments in zebrafish that had developed granulomas, the hallmark of tuberculosis, ${ }^{28,29}$ and showed that polymersomes penetrate such environment, which is hard to access. ${ }^{30,31}$ These evidences led us to investigate the potential of antimicrobial-loaded polymersomes for intracellular pathogens clearance, both in vitro and in vivo. We demonstrated that PMPC-PDPA polymersomes, loaded with anti-mycobacterial drugs (gentamicin, lysostaphin, vancomycin, rifampicin, and isoniazid), are able to decrease, and in some cases even eradicate, intracellular S. aureus, M. bovis-attenuated Bacillus Calmette-Guérin (BCG), M. Marinum and M. tuberculosis.

\section{Results and discussion}

\section{Polymersomes drug release profile and internalization dynamics in human macrophages.}

The selective targeting of specific immune cells sub-populations represents a new paradigm in precise nanomedicine and will have huge impact in fields like cancer immunotherapies or infectious diseases. We explored in this work the possibility of targeting the immune system and studied how polymersomes can be used to deliver drugs for the treatment of intracellular pathogens, which are more difficult to eradicate compared to extracellular bacteria.

To do this, we synthesized PMPC-PDPA copolymers using atom transfer radical polymerization (ATRP), and fully characterized the products of the reaction by gel permeation chromatography (GPC) (Figure S1a) and NMR spectroscopy (Figure S1b). PMPC-PDPA was also functionalized with Cy5 dye by click reaction to produce fluorescent polymers. Then, we used the film hydration method to induce the self-assemble of the PMPC-PDPA copolymers into vesicles of about $100 \mathrm{~nm}$. It is worth mentioning that film hydration usually induces the formation of differently shaped nanostructures. We thus isolated monodisperse spherical polymersomes by means of density gradient centrifugation. ${ }^{32}$ Transmission electron microscopy (TEM) confirmed the purification processes were successful in isolating spherical polymersomes with homogeneous shape distributions (Figure Sc1 and Sc2). The encapsulation of drugs did not affect polymersomes shape (Figure Sd1 and Sd2) nor changed their size distribution (Figure S1f). Also, the polymer 
functionalization with Cy5 was confirmed to be stable under harsh acidic condition ( $\mathrm{pH} 2$ ), a crucial aspect for correct cell uptake quantifications (Figure S1e). We then studied the drug release profile of $\mathrm{pH}$ sensitive PMPC-PDPA polymersomes during time. Free- and rifampicin-loaded polymersomes have been placed in a dialysis bag under stirring (see materials and methods section for details). Rifampicin-loaded polymersomes do not release the drug at physiological $\mathrm{pH}(\mathrm{pH} 7.4)$, confirming the high stability in circulation-like conditions (Figure S2, pink line). The drop in $\mathrm{pH}$ ( $\mathrm{pH}$ 6) triggers a steady release of the encapsulated drug (Figure S2, red line). Conversely, there is no control over free (non-encapsulated) rifampicin distribution, which just follows its gradient concentration equilibrium (Figure S2, blue and cyan lines). This is a quite important outcome, as polymersomes release the drug only upon internalization in the cells and avoid undesired drug distribution in other body compartments. At the same time, the slow polymersomes-driven release (c.a. $20 \%$ of the initial dose) makes them a powerful drug reservoir that is constantly and steadily released in the cells for long times.

We then studied the kinetics of PMPC-PDPA polymersomes internalization in vitro in macrophages using the monocytes-derived macrophages THP-1. Live cell confocal laser scanning microscopy (CLSM) imaging of macrophages stained by CellMask ${ }^{\mathrm{TM}}$ shows that the uptake of PMPC-PDPA polymersomes occurs within minutes post-exposure (Figure 1a). We observed full saturation of the membrane within minutes after incubation, with several internalization events occurring few seconds after the initial contact of the polymersomes with the plasma membrane. The kinetic plots of uptake for four regions of interest (ROI) confirm rapid binding and endocytosis with very little difference between the plasma membrane or cytosolic ROIs (Figure 1b). Representative confocal 3D scans (Figure 1c) show the presence of the polymersomes within the entire volume of the macrophages. We then addressed the intracellular trafficking of polymersomes. It is important mentioning that THP-1 macrophages are challenging to be transfected with external genetic materials. Hence, it is very difficult to create chimera proteins (e.g., GFP-fusion) with the aim to carry out live cell imaging of (marked) intracellular organelles, and their possible co-localization with polymersomes. For THP-1, the two possible options are thus post-fixation methods like immunofluorescence or live imaging based on chemical staining (e.g., lysotracker). Cy5-labelled polymersomes have been incubated with macrophages for a short period of time (30 minutes). Immunofluorescence analyses (Figure 1d) show no co-localization signal between the Cy5polymers and the early endosome antigen 1 EEA1 (which marks the early endosomes). Similar results were observed during live-cell imaging, where THP-1 cells were first incubated with Cy5polymersomes again for 30 minutes and then stained with LysoTracker (for marking all the acidic compartments). The lack of co-localization suggest that the Cy5-polymers diffuse out of the endocytic pathways. We then speculated that the slow sustained PMPC-PDPA drug release (Figure S2) could induce accumulation of polymersomes in later stages of endocytosis as a function of both (high) concentration of polymersomes and incubation time. We tested this hypothesis and confirmed that Cy5-polymers accumulate in the lysosomal compartments after very long incubation time (24 and 72 hours, Figure S3a-b). The question of timing is indeed an important aspect to boost the amount of drug in the compartments where bacteria hide and proliferate. We also CLSMimaged and quantified the presence of polymersomes at 8, 24 and 72 hours of incubation time (Figure S4a). Calcein (green) staining further validated the efficient uptake and intracellular distribution of polymersomes by macrophages, which remained viable for the incubation time tested. We further quantified the uptake using high performance liquid chromatography (HPLC) of the cell lysates after different incubation times. HPLC-based uptake quantifications revealed about $10^{4}$ polymersomes/cell after 8 hours, the number of polymersomes rose by $3 \cdot 10^{4}$ after $24 \mathrm{~h}$ and this remained constant up to 72 hours of incubation (Figure $\mathrm{S} 4 \mathrm{~b}$ ). 

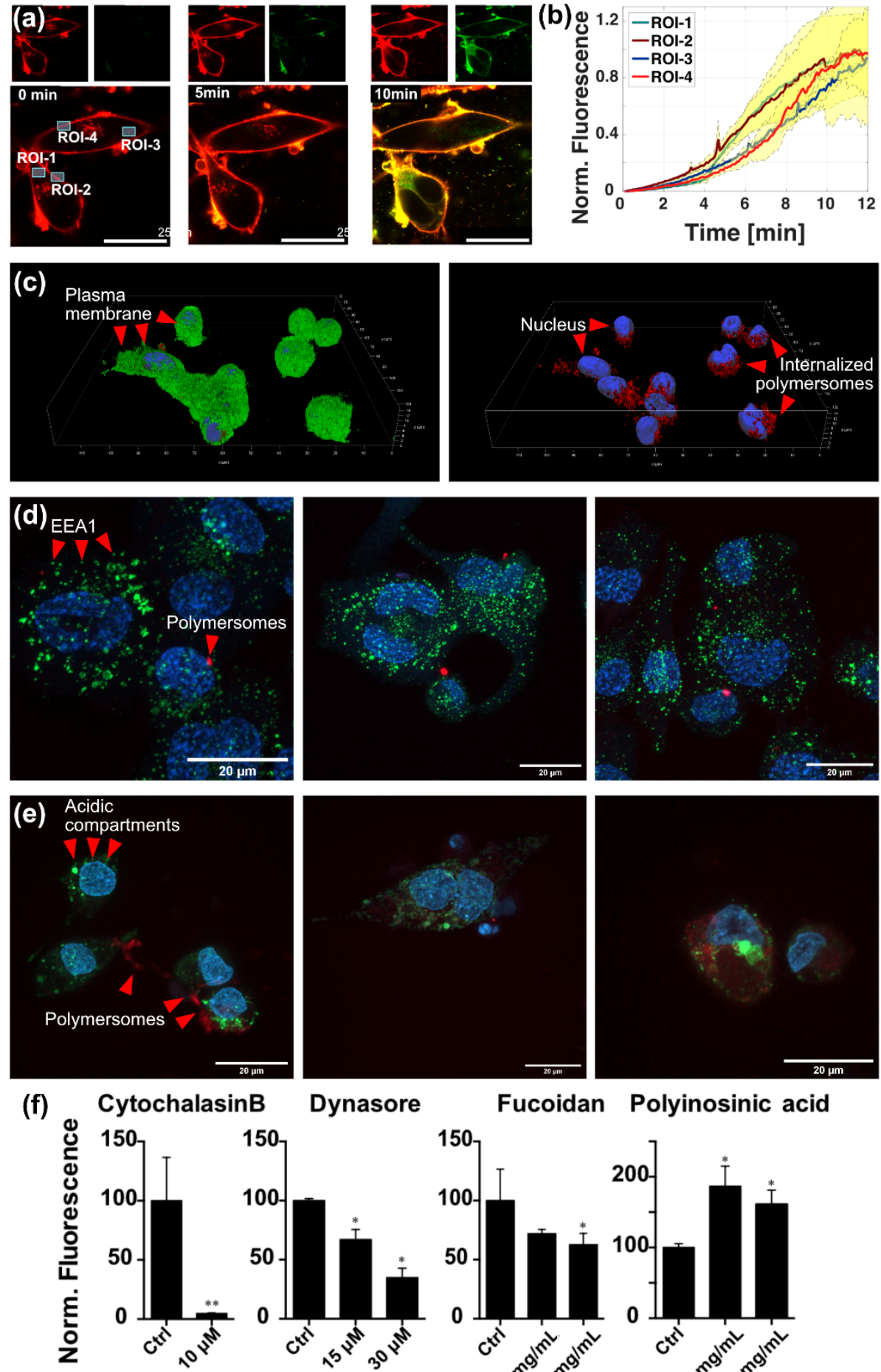

Fucoidan Polyinosinic acid
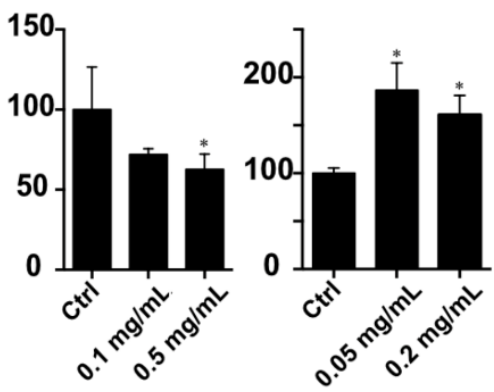

Figure 1. PMPC polymersomes interaction with phagocytes in vitro. (a) Real-time imaging of polymersomes entering monocyte-derived macrophages (THP-1 cells) using confocal laser scanning microscopy (CLSM). Note the polymersomes (red signal) are labelled using Cy5, and the macrophage membrane (green signal) is stained using CellMaskTM. (b) Polymersomes uptake measured in 4 different regions of interest (ROI) in (a) plotted as a function of time. (c) Confocal 3D scan of THP-1 cells incubated with Cy5 polymersomes. (d) Immunofluorescence analyses showing no co-localization between polymersomes (red) and EEA1 (green). (e) Live cell imaging of polymersomes (red) and LysoTracker-stained (green) cells. (f) Polymersomes uptake after inhibition of different cellular components: CytochalasinB (actin inhibitor), Dynasore (dynamin inhibitor), Fucoidan (Scavenger Receptors A and B inhibitor), and Polyinosinic acid (Scavenger Receptor A inhibitor and Toll-like 3 receptor agonist ligand stimulator). ( $t$-test comparison with $\left.{ }^{*} p<0.05\right)$. 
We then investigated the driving force for polymersomes internalization and studied the receptors involved in their uptake. To investigate this, macrophages were incubated with the actin inhibitor cytochalasinB. We observed a complete inhibition of polymersomes uptake, confirming that the entry process is mediated by actin-dependent transport (Figure 1f). Moreover, incubation with $15 \mu \mathrm{M}$ and $30 \mu \mathrm{M}$ dynasore (a dynamin inhibitor) reduced the polymersomes uptake by $40 \%$ and $60 \%$, respectively, but did not stop it completely (Figure 1f). The GTPase dynamin regulates membrane fission in clathrin-mediated endocytosis, as well as in phago- and macropinocytosis in eukaryotic cells. ${ }^{33}$ Few dynamin-independent entry pathways have been described, and they include the CDC42 (the preferred entry route of cholera toxin B), ${ }^{34} \mathrm{ARF} 1$ and ARF6, ${ }^{35,36}$ and Flotillin 1 and 2 pathways. ${ }^{37,38}$ The polymersomes uptake in the presence of dynasore suggest that they can gain access through dynamin-independent endocytosis. Scavenger Receptors (SR) and SR-B1 in particular, are known receptors for PMPC-PDPA polymersomes uptake in non-professional phagocytes. ${ }^{23} \mathrm{SR}-\mathrm{B} 1$ is known to play an critical role in pathogens recognition and in cholesterol homoeostasis. ${ }^{39-41}$ To test whether macrophages also internalize polymersomes using scavenger receptors, we incubated the cells with fucoidan, an inhibitor of Scavenger Receptors class A and B (SR-A and B). Despite the presence of the inhibitor, polymersomes were able also in this case to access macrophages, albeit with a considerable decrease in uptake of about $40 \%$ (Figure 1f). In order to define the contribution of the class A or B, macrophages were treated with polyinosinic acid (PA), a selective inhibitor of SR-A. Surprisingly, PA led to a significant increase in polymersomes uptake (Figure $2 \mathrm{~b}$ ), even though this can be explained by the fact that PA can improve uptake activities by binding to Toll-like receptor $3 .{ }^{42}$ The inhibition studies, albeit not conclusive about the exact endocytic process involved, suggest that polymersomes uptake is a complex orchestra of multiple pathways.

\section{Polymersomes are safe delivery agents}

Even though many polymersomes were internalized by the macrophages, viability assays (MTT) confirmed that free- and antimicrobial-encapsulated polymersomes do not affect the metabolic activity of THP-1 cells for concentrations up to $1 \mathrm{mg} / \mathrm{mL}$ (Figure 2a). To provide a broader overview of potential change in cell metabolism, we investigated the expression of specific stressrelated genes upon polymersomes incubation. We quantified the expression profiles of the (i) $p 21$ and $p 53$ genes, key regulators of cell cycle and apoptosis, of the (ii) superoxide dismutase (SOD) and catalase (CAT) to check for potential $\mathrm{O}_{2}$ radicals-induced oxidative stress (likely to occur in macrophages), and of the (iii) detoxification-related cytochromes CYP1Aq and CYP1B1. We also quantified the expression levels of ATF4 and ATF6, which are the sensors of the unfolded protein response pathway (UPR). UPR is an adaptive cellular program used by eukaryotic cells to cope with protein misfolding stress. We thus covered a broad panel of stress-related pathways and did not detect any significant differential regulations of genes between untreated- and polymersomestreated macrophages (Figure 2b). We confirmed that high amount of polymersomes $(1 \mathrm{mg} / \mathrm{mL})$ did not trigger any inflammation in macrophages as well. This has been validated by immunofluorescence analyses, where the localization of the transcription factor nuclear factor $\mathrm{\kappa B}$ (Nf- $\mathrm{kB}$ ) has been assessed (Figure 2c-f). It is indeed established that in non-inflamed conditions, the majority of the Nf- $\kappa \mathrm{B}$ is localized within the cytosol, while higher nuclear presence of $\mathrm{Nf}-\kappa \mathrm{B}$ indicates that macrophages are triggering inflammation. Being a transcription factor, the Nf- $\mathrm{kB}$ promote the expression of a whole panel of pro-inflammatory cytokines that regulate the inflammation process. Our data show that macrophages treated with polymersomes (Figure 2d) have the same nuclear presence of Nf- $\kappa \mathrm{B}$ of untreated cells (Figure 2c), while lipopolysaccharide (LPS)-treated macrophages have a significantly higher nuclear presence of the transcription factor (Figure 2e). The co-localization quantification (Figure $2 \mathrm{f}$ ) confirms the safety of polymersomes. 
(a)
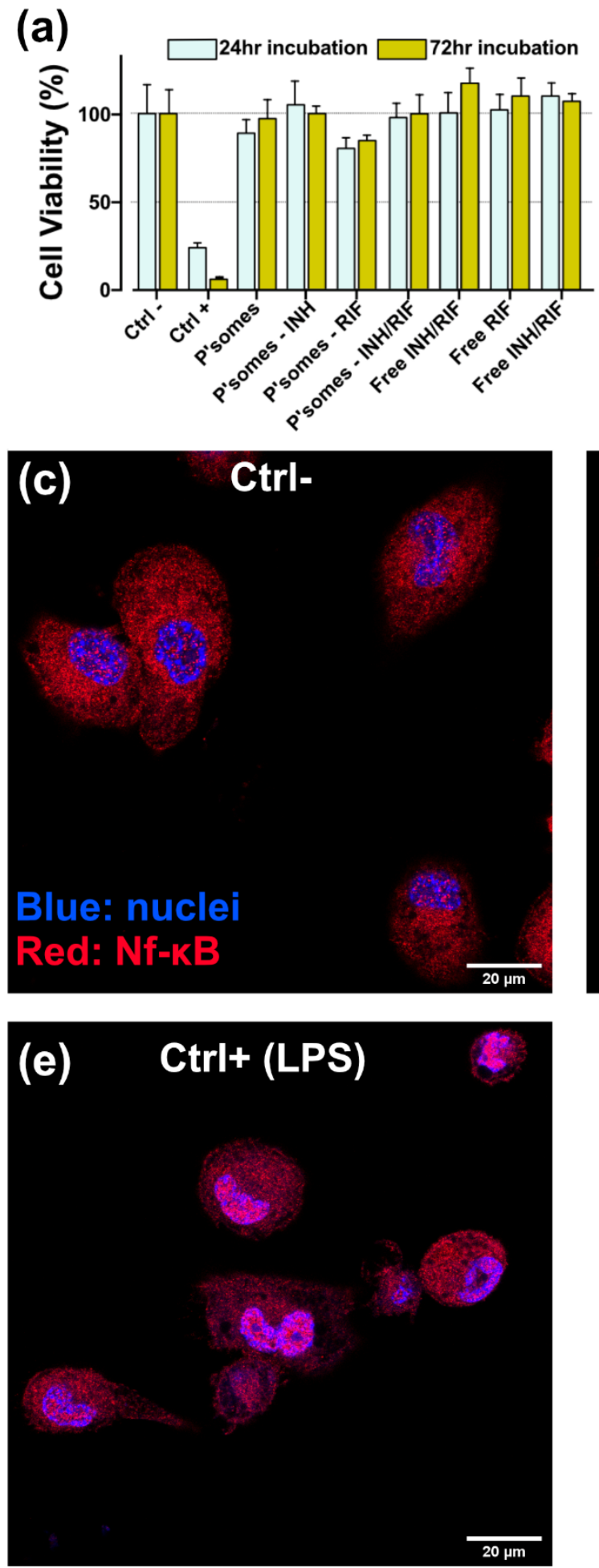

(b)

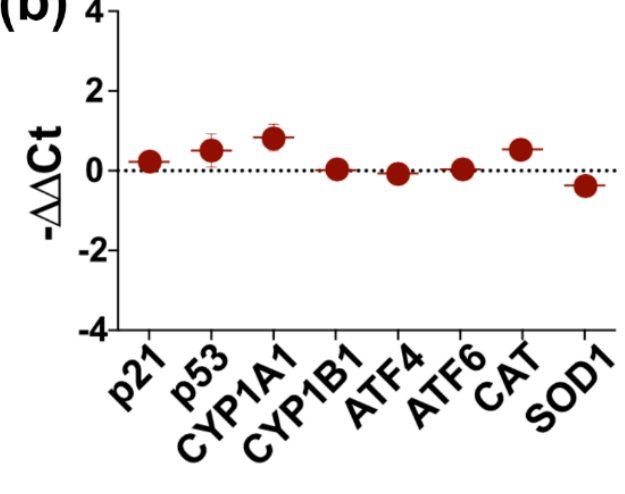

(d) Polymersomes

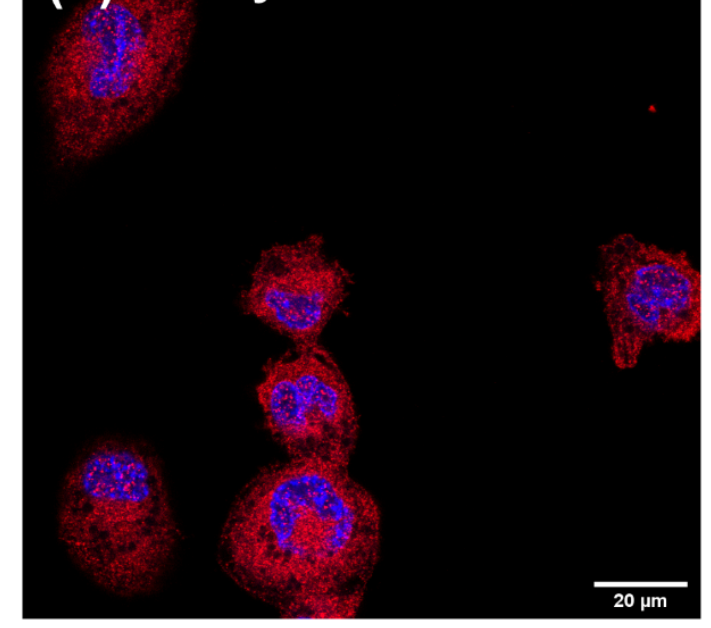

(f)

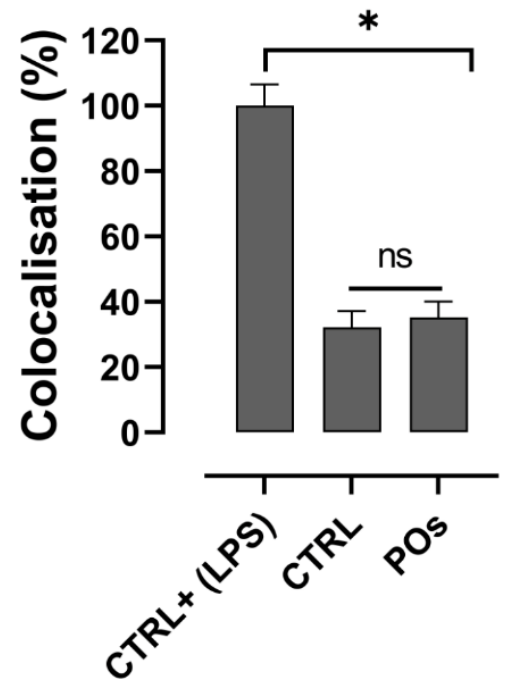

Figure 2. Polymersomes biocompatibility. (a) Viability assays (MTT) of THP-1 cells incubated with un-loaded and with antibiotic-loaded (rifampicin, isoniazid, and combination of both) polymersomes. Ctrl-: Cells treated with PBS; Ctrl+; DMSO 5\%; [polymersomes]: $1 \mathrm{mg} / \mathrm{mL}$; [RIF]: $30 \mu \mathrm{g} / \mathrm{mL}$; [isoniazid]: $3 \mu \mathrm{g} / \mathrm{mL}$. (b) Quantitative PCR (qPCR) for analyzing the expression levels of genes involved in cell proliferation (p21 and p53), cell stress (CYP1A1 and CYP1B1), Unfolded Protein Response (ATF4 and ATF6), and oxidative stress (CAT and SOD). (c-e) Representative immunofluorescence imaging to assess $\mathrm{Nf}$ - $\mathrm{kb}$-based inflammation in untreated- (c), polymersomestreated (d), and LPS-treated (e) macrophages. Red: Nf-kb, blue: nucleus. (f) Co-localization quantification of the images in (c-e) using the Pearson's correlation coefficient. 


\section{Biodistribution of polymersomes in zebrafish}

We moved to a relevant in vivo model and chose the Danio rerio (zebrafish) embryo. These animals are optically transparent, allowing observation of polymersomes targeting and delivery over time in the same animal. The availability of fluorescent transgenic lines labelling immune cell populations allows imaging of macrophages and neutrophils. ${ }^{43}$ Furthermore, there are wellestablished zebrafish models of human-relevant infections of $S$. aureus, ${ }^{44,45}$ and M. marinum (a close relative of human TB complex, and a natural pathogen of fish species). ${ }^{46-50}$ In order to evaluate the potential of the polymersomes to target intracellular pathogens we tested the nanoparticles in zebrafish infected with M. marinum, the causative agent of tuberculosis in ectotherms and a close relative of M. tuberculosis. For this, about 200 mycobacteria expressing GFP were injected intravenously (blood infection) at day 2 post fertilization in the recombinant line of zebrafish Tg (mpeg 1:mcherry), which has macrophages fluorescently labelled (Figure 3a). After 24 hours, we injected Cy5 labelled polymersomes and monitored their uptake by macrophages as a function of time. Polymersomes could be observed within the target cells already 10 minutes after injection, and their intracellular uptake increased over the following 24 hours (Figure 3b). At 8 hours post injection, polymersomes clearly aggregated into macrophages already infected with $M$. marinum (Figure 3a, c and d). Supporting Video 1 and Figure S7 show in detail the intracellular localization within macrophages of both polymersomes and M. marinum. We quantified the caudal region of 3 zebrafish larvae and observed polymersomes within $93 \%$ of their intended targets (40 out of 43 M. marinum-infected macrophages). Qualitative images also show that polymersomes are taken up by infected macrophages already at 10 minutes and persist up to three days in individually infected macrophages (Fig S5a-d). Moreover, polymersomes were seen surrounding and then penetrating not only individual macrophages but also the first macrophage aggregates (early granulomas) that form by three-day post infection (Figure Sd1-d3). At the same time, neutrophils were not targeted by the same polymersomes, demonstrating a high level of selectivity towards macrophages only (Figure S6). Similar results were obtained when the infecting agent of zebrafish was $S$. aureus (Figure S5e,f). In this case, fluorescent lysostaphin delivered by polymersomes into infected phagocytes co-localized with intracellular $S$. aureus (Figure S5f). To evaluate if polymersomes can enter bigger granulomas, we employed a different model of $M$. marinum where the pathogen is injected directly into the neural tube of the zebrafish at day 3 post birth (Figure 3e). This type of infection promotes the formation of large granulomas made of hundreds of cells, a hallmark of tuberculosis disease. Polymersomes injected intravenously at day 4 post infection clearly localize within the granuloma after 8 hours (Fig 3e-g and Supporting Video 2). Image analysis confirmed that polymersomes quickly accumulated within the granulomas during the first hour post administration and continued to further concentrate, albeit slower, over time (Figure $3 \mathrm{~h}$ ). This new zebrafish neural tube infection model developed here has the advantage of possessing some of the characteristics missing in the mouse granulomas, which are present in human TB. These features are local necrosis, vascular thrombosis, cavity formation and hypoxia. ${ }^{51}$ Also, there are evidence that zebrafish can be efficiently used to predict the circulation of nanoparticles in mice. It thus represents a valid alternative to mammalian models for pre-clinical screenings. ${ }^{52}$

\section{Efficacy of polymersomes in eradicating intracellular bacteria}

After assessing the polymersomes distribution at cellular level and in zebrafish, we moved on addressing their efficacy in reducing bacterial burden in infected cells. We used the model intracellular pathogens S. aureus, M.bovis-BCG, M. tuberculosis, and M. marinum. First, we confirmed that polymersomes can effectively load vancomycin, gentamicin, lysostaphin, rifampicin and isoniazid (Figure 4a). To this respect, it is important to mention that the different drugs have considerable differences in molecular mass, hydrophilicity, and mechanism of action. 

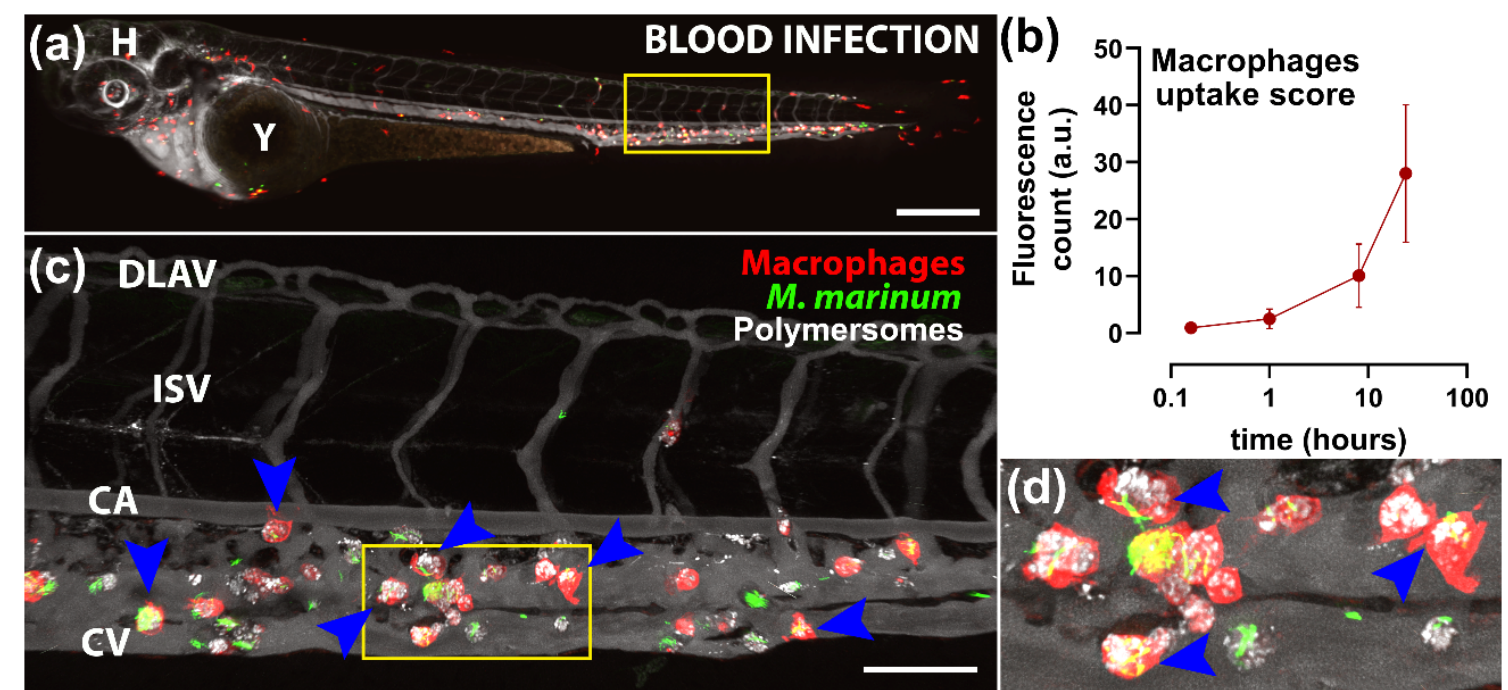
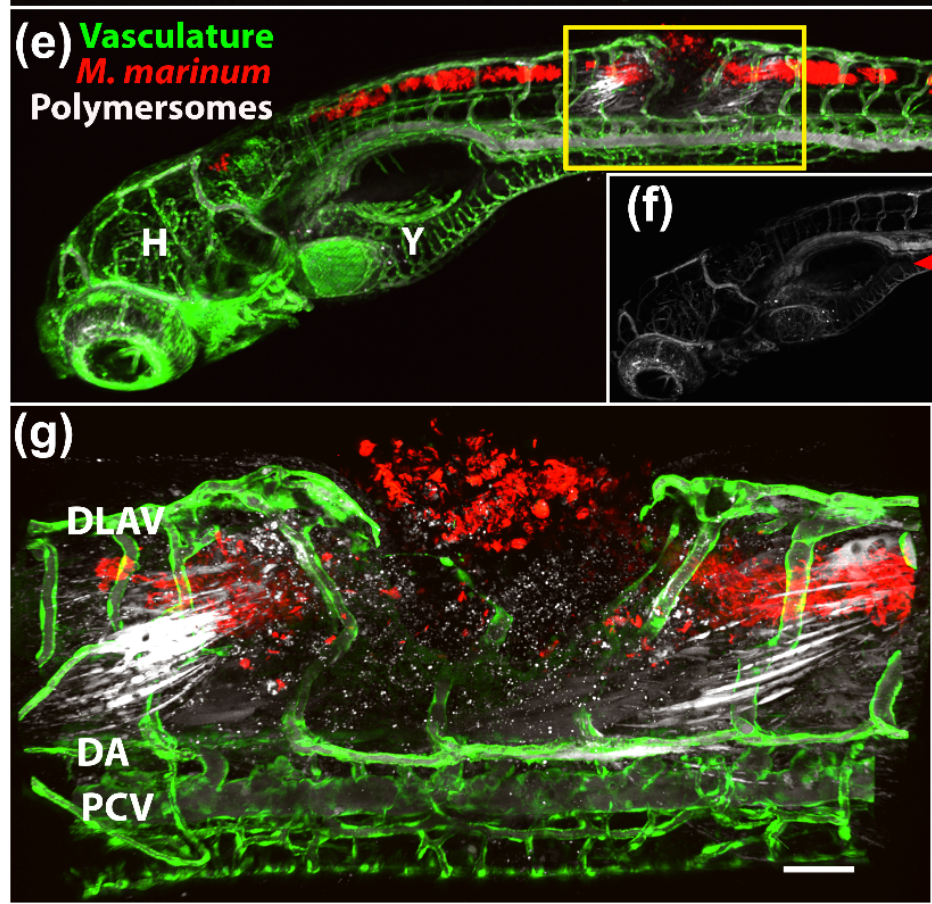

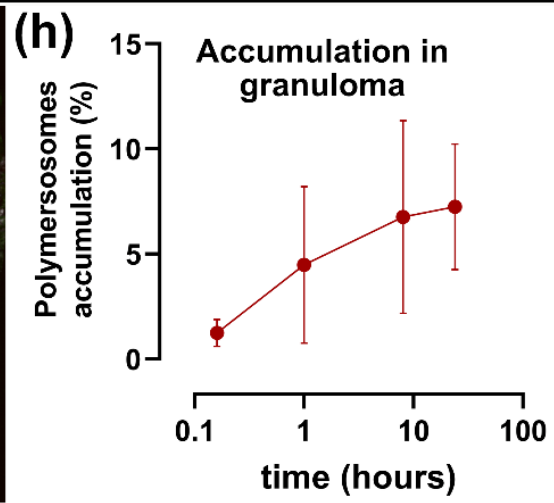

Figure 3. Polymersomes accumulation in macrophages and granulomas in zebrafish embryos infected with M. marinum. (a). A zebrafish embryo fluorescently labelled with macrophages (red) injected intravenously with M. marinum and the following day with polymersomes containing Cy5 (white). The image was taken 8 hours after polymersomes injection. H, head region; Y, Yolk sac. (b) Quantification of polymersomes uptake over-time in macrophages in zebrafish larvae. (c) enlarged area in (a) where polymersomes are detected within infected macrophages (blue arrows). DLAV, Dorsal longitudinal anastomotic vessel. ISV, intersegmental vessel; CA, Caudal Artery; CV, Caudal Vein. (d) enlarged area in (c) where macrophages containing M. marinum and polymersomes are evident (blue arrows). (e) Zebrafish embryos fluorescently labelled with endothelial cells (green) were injected with $M$. marinum (red) in the neural tube. Four days later, polymersomes containing Cy5 (white) were injected intravenously and the whole zebrafish was imaged eight hours later. H, Head region; Y, Yolk sac. (f) shows the image in (e) without the signal of green endothelial cells and red M. marinum in order to better observe the selective accumulation of polymersomes (blue arrows) in the granuloma region. The yellow box in (e) is seen enlarged in (g) for observing details of polymersomes accumulation in the granuloma. DLAV, Dorsal Longitudinal Anastomotic Vessel; DA, Dorsal Aorta; PCV, Posterior Cardinal Vein. A graph showing accumulation over time of polymersomes in neural tube granulomas is shown in (h). Scale bars: (a), $300 \mu \mathrm{m}$; (c) $100 \mu \mathrm{m}$, (d) $25 \mu \mathrm{m}$. (e), $300 \mu \mathrm{m}$, (f), $300 \mu \mathrm{m}$, (g), $50 \mu \mathrm{m}$. 
Lysostaphin is a $27 \mathrm{KDa}$ glycylglycine endopeptidase only soluble in water acting on the $S$. aureus cell walls. Gentamicin is a highly hydrophilic aminoglycoside that binds to the 30S subunit of the bacterial ribosome. Vancomycin is a relatively hydrophilic glycosylated non ribosomal peptide that inhibits cell wall synthesis. Rifampicin is a hydrophobic heterocyclic modified naphthoquinone that inhibits bacterial DNA-dependent RNA synthesis. Isoniazid is a small synthetic derivative of nicotinic acid with a poor water solubility that upon enzymatic activation inhibits the synthesis of mycoloic acids. These drugs are used clinically for the treatment of several infections and make a very diverse population of molecules to test the versatility of polymersomes. We tested the effect of the antimicrobials in the treatment of different infections by measuring the colony forming units (CFUs) after increasing incubation periods. Treatment with polymersomes loaded with rifampicin or gentamicin improved the drug efficacy and reduced the number of viable $S$. aureus in THP-1 cells compared with controls (Figure 4b). Encapsulation of lysostaphin or vancomycin within polymersomes did not significantly improve or hinder drug efficacy. For both BTG and M. tuberculosis, we limited our screening to rifampicin and isoniazid either alone or in combination mirroring the most common therapeutic approach used for the treatment of tuberculosis. With respect to BCG infection, no significant differences were observed in CFUs after 1 day of treatment (Figure 4c). Only the free rifampicin was able to reduce the bacterial colonies. However, a significant difference was observed after 72 hours of treatment, where both rifampicin and isoniazid-encapsulated polymersomes elicited a clear reduction in bacteria compared to the free drug (Figure 4c). Notably, the rifampicin/isoniazid co-loaded polymersomes completely eradicated the intracellular BCG after 72 hours (no CFUs detected). Similar results were observed with M. tuberculosis infected THP-1 cells (Figure 4d). In this case, after 24 hours of treatment, the multiple drug co-loaded polymersomes significantly reduced bacterial burden compared to the controls. Moreover, this drug formulation was also able to eradicate intracellular M. tuberculosis after 72 hours of treatment (Figure 4d). The intracellular CFUs are very-well known to normally rise inside host macrophages if no antimicrobials are inoculated ${ }^{53,54}$ It is important mentioning that an improvement in polymersomes-mediated delivery was not detected only upon using lysostaphin (against $S$. aureus) and isoniazid (against M. tuberculosis). Two possible hypotheses can be inferred to explain this behavior. First, the drug mechanism of action can be altered by the cytosolic environment. Second, both endo-lysosomal and cytosolic-resident bacteria are targeted. This can have significant different outcomes depending on the specific drug used. For example, while there was not any improvement for isoniazid treating M. tuberculosis, a one log improvement was observed for isoniazid treating M. bovis. This suggests that $M$. tuberculosis is more efficient in escaping into the cytosol. ${ }^{55}$ Also, isoniazid may act better within endo-lysosomal compartment rather than within the cytosol. Most importantly, the combination isoniazid/rifampicin is the most successful with full sterilization in M. tuberculosis infected macrophage.

We finally validate the therapeutic impact also in zebrafish and tested the ability of polymersomes encapsulated antibiotics to reduce bacterial burden in vivo. Zebrafish embryos were infected with mCherry-expressing M. marinum, and with GFP expressing $S$. aureus. In the $S$. aureus infection model, zebrafish received an injection of $1200 \mathrm{CFU}$, at which dose the infection is either cleared or leads to rapid death of the fish. Zebrafish begin to succumb to the infection after approximately 40 hours post infection, so this time-point was used as an output to determine the extent of zebrafish infection. To compare the effect of encapsulated antimicrobials and free antimicrobials to treat $S$. aureus infection, zebrafish embryos were injected with $S$. aureus followed by a second injection of drug loaded polymersomes 20 hours later. We assessed the efficacy of the four drugs tested in vitro, lysostaphin, vancomycin, gentamicin and rifampicin (Figures 5a-b). In agreement with the in vitro results, only encapsulated rifampicin and gentamicin treatment improved the outcome of infection. 

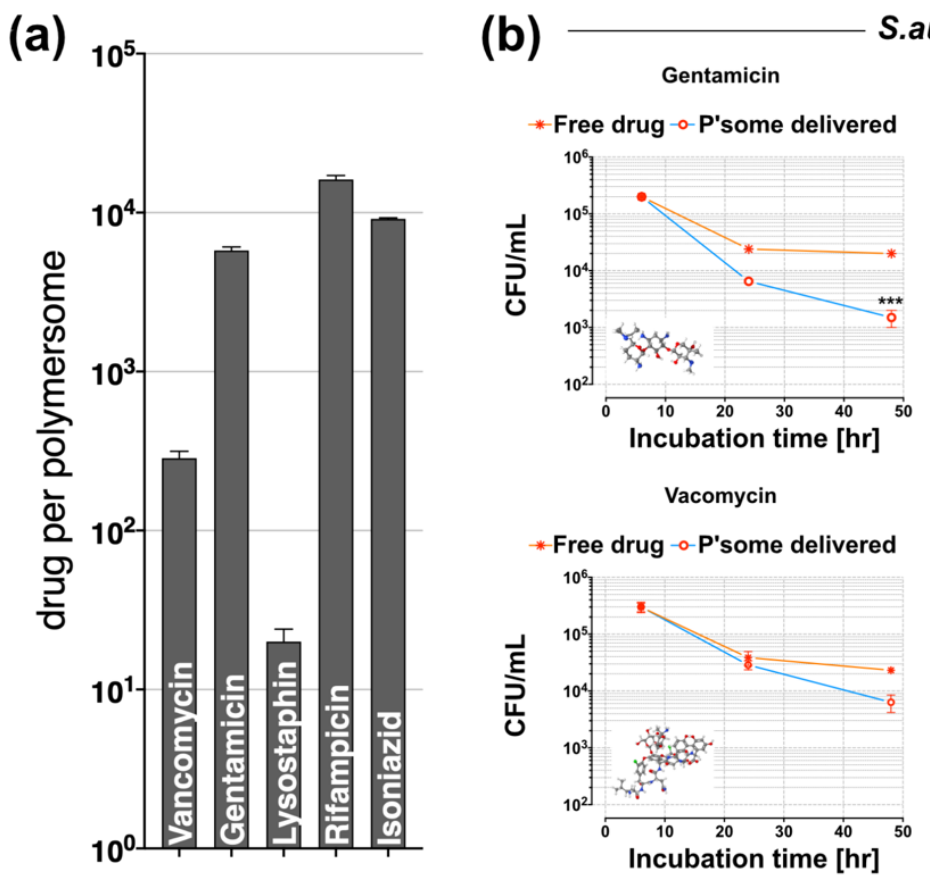

S.aureus
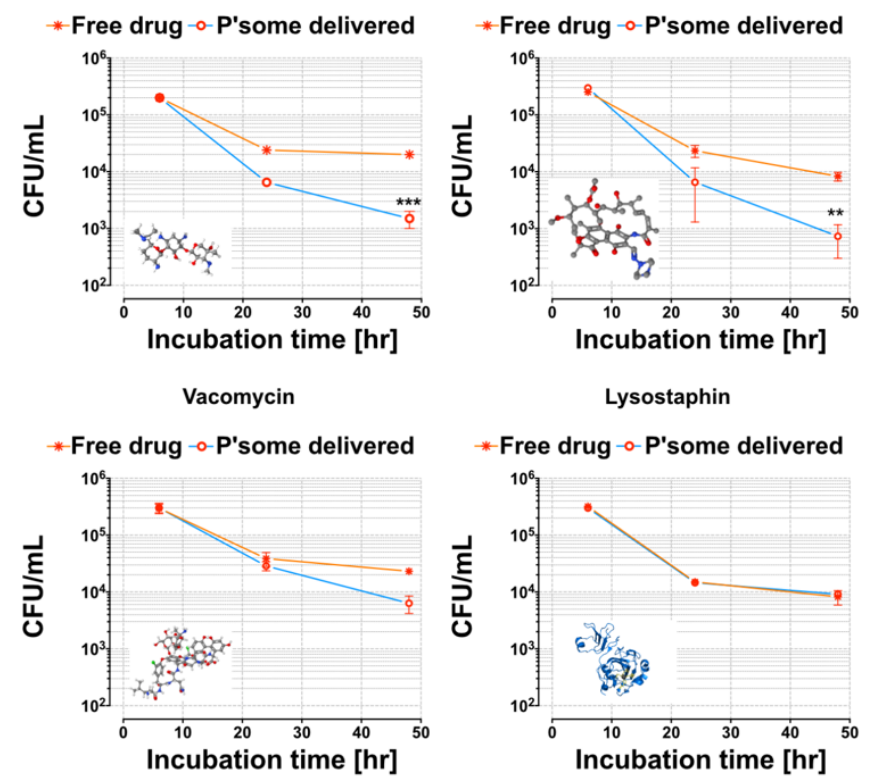

Lysostaphin

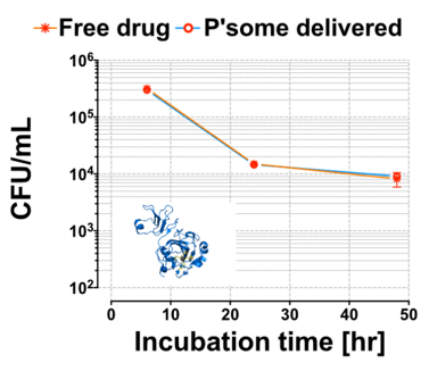

(c)

M.bovis (BCG)
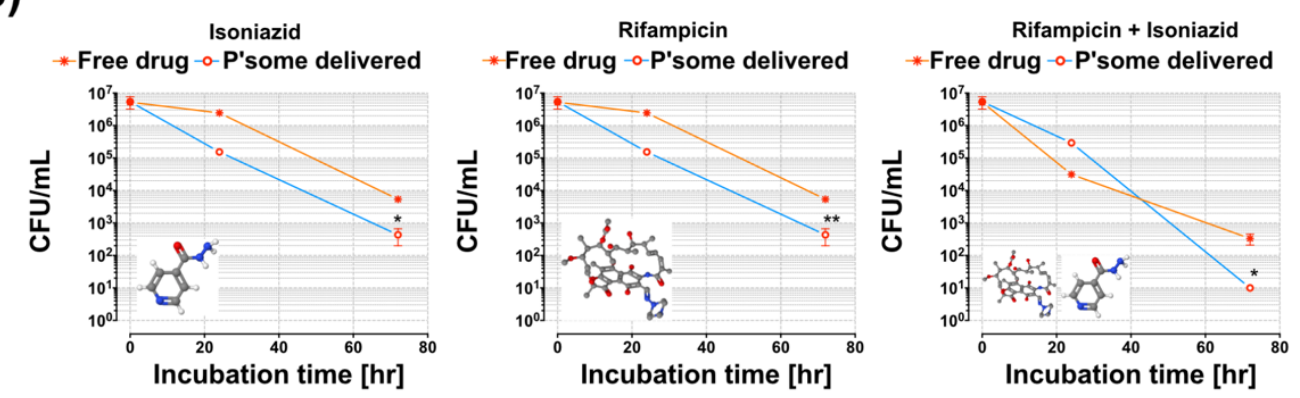

(d)

M.tuberculosis
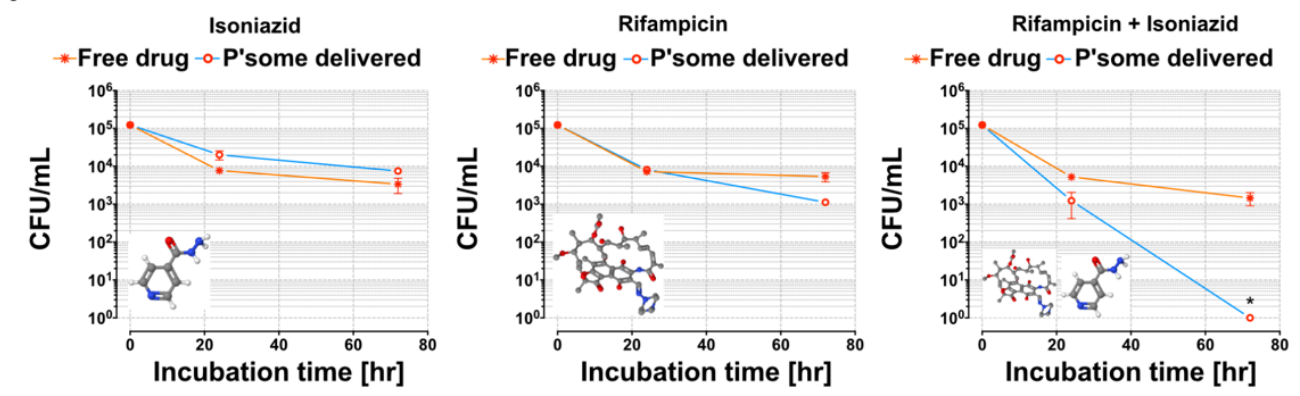

Figure 4. Polymersomes eradicating intracellular pathogens in human macrophages. (a) Average number of drug per single polymersome measured by HPLC (b) THP-1 macrophages infected with $S$. aureus (M.O.I of 5:1) for 6 hours. Following infection, gentamicin was added to the media to kill extra-cellular bacteria. Macrophages were subsequently treated with polymersomes encapsulating gentamicin, rifampicin, vancomycin, or lysostaphin (all at $1 \mu \mathrm{g} / \mathrm{mL}$ ). At 6, 22, and 46 hours macrophages were lysed and plated on a BHI agar plate for bacterial colonies to be counted (One-way ANOVA $* * p<0.01, * * * p<0.001$, error bars $=$ SEM, $\mathrm{n}=3$ ). Viability (CFU) analyses of BCG (c), and (d) M. tuberculosis after 24 and 72 hours of incubation with the different formulations (One-way ANOVA with *p<0.05and $* * p<0.01$ ). 
Lysostaphin and vancomycin did not change the outcome of infection, with similar numbers to the control groups showing high numbers of bacteria (Figure 5a). The polymersomes-encapsulated rifampicin was the most effective treatment, resulting in a reduction in the bacterial CFUs and preventing the fish from succumbing to overwhelming infections. Polymersomes did improve considerably the output with the rifampicin formulation getting very low CFUs and with survival close to $100 \%$. The efficacy of polymersomes delivered rifampicin was confirmed using a second in vivo model, the $M$. marinum infected zebrafish model of TB. In this case, mCherry-expressing

(a)

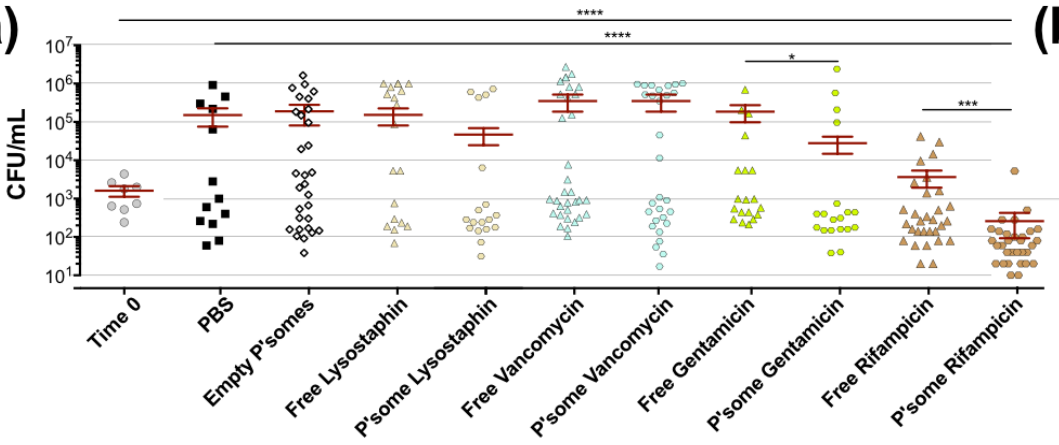

(b)

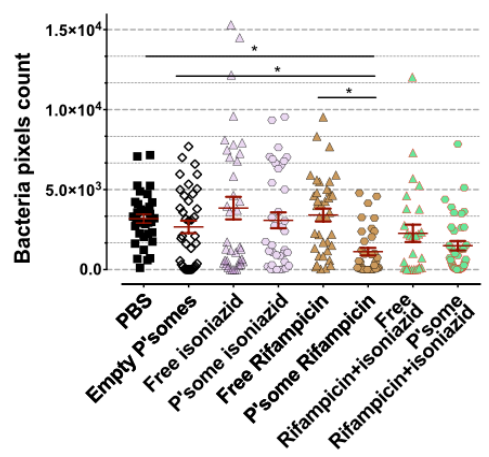

Figure 5. Enhanced efficacy of antimicrobials in vivo upon encapsulation in polymersomes. (a) Zebrafish embryos 2 days post infection were injected with $S$. aureus (data time 0 ) followed by a second injection 20 hours later with either PBS, empty polymersomes, free drug and polymersomes loaded with lysostaphin, vancomycin, gentamicin, and rifampicin. Zebrafish were then left for 20 hours before being homogenized and plated on BHI agar for viable colony counts. Graphs show the total number of CFU after treatment (Kruskal-Wallis test with Dunn's multiple comparison $* p<0.05, * * p<0.01$ and $* * * p<0.001$ ). (b) Quantification of mCherry expressing $M$. marinum bacterial burden in zebrafish embryos treated with empty polymersomes, free drugs, and polymersomes loaded with rifampicin, isoniazid, and their combination. (ANOVA test comparison with $* p<0.05)$.

fluorescent bacteria were microinjected, and 24 hours later an injection of the polymersomesencapsulated drugs (or controls) was performed. As was the case for $S$. aureus infected zebrafish, rifampicin-encapsulated polymersomes significantly reduced the M. marinum burden in vivo, compared to the same concentration of free drug (Figures $5 \mathrm{~b}$ ). The data on zebrafish point out two main questions. First, there is a good matching between the in vitro and in vivo assays, which is a fundamental validation of the data. Second, we confirmed that much lower doses of drugs are required to reach a therapeutic concentration inside the cells when the antimicrobials drugs are encapsulated. This, in turn, can significantly contribute to the reduction of antimicrobial drug resistance.

\section{Conclusions}

Finding alternative and more effective solutions to bacterial infections is becoming increasingly important with the rise of antimicrobial resistant bacteria rendering many therapies ineffective. In addition, serious diseases like TB require long-term treatments, which usually need doses of a combination of antibiotics for long periods (at least six months), with a consequent rise of serious side effects, and bacterial resistance. New therapies, which can selectively target only infected 
phagocytes, are nowadays required in order to improve the efficacy while limiting off target side effects. In this work, we have demonstrated that PMPC-PDPA polymersomes can be loaded with a large variety of antibiotics, including proteins (lysostaphin), small peptides (vancomycin), glycols (gentamicin), poorly water-soluble organics such as quinones (rifampicin) and functionalised pyridines (Isoniazid), thus covering a large repertoire of possible chemistries. We have shown that polymersomes can deliver antibiotics to treat intracellular pathogen-related infections, and to potentially decrease the dose and duration of treatment required for bacterial eradication. Both in vitro in human cells and in vivo experiments demonstrated that these nanoscopic synthetic vesicles were internalised by macrophages, without inducing toxicity, and were able to escape the endocytic pathway. We have demonstrated that drug-encapsulated polymersomes were able to reduce $S$. aureus, BCG, M. tuberculosis, and $M$. marinum bacterial burden, again using in vitro and in vivo approaches. Antimicrobial-loaded polymersomes were more effective compared with the same concentration of free drug, and in some cases were able to eradicate the intracellular microorganisms completely. We thus believe this technology can be exploited to reduce the effective dose required for therapy, with a consequent potential reduction in antimicrobial resistance. In addition, encapsulation of antimicrobials could help completely eradicate infection from the host more rapidly, by direct delivery of drug to the immune system to enhance the hostpathogen response.

\section{Materials and Methods}

PMPC-PDPA copolymer synthesis. In a typical ATRP procedure, a $100 \mathrm{~mL}$ round bottom flask equipped with a magnetic stir bar and a rubber septum was loaded with 2-methacryloyloxyethyl phosphorylcholine (MPC, $5 \mathrm{~g}, 16.9 \mathrm{mmol})$, 2-(4-morpholino)ethyl 2-bromoisobutyrate (ME-Br) initiator $(189 \mathrm{mg}, 0.7 \mathrm{mmol})$ and $6 \mathrm{~mL}$ ethanol, and this solution was deoxygenated by purging $\mathrm{N}_{2}$ for $1 \mathrm{~h}$ under stirring at room temperature. Then, 2,2'-bipyridine (bpy) ligand (212 $\mathrm{mg}, 1.4 \mathrm{mmol}$ ) and $\mathrm{Cu}(\mathrm{I}) \mathrm{Br}(97 \mathrm{mg}, 0.7 \mathrm{mmol})$ were added as solids whilst maintaining the flask under a mild positive $\mathrm{N}_{2}$ pressure. The [MPC]:[ME-Br]:[CuBr]:[bpy] relative molar ratios were 25:1:1:2. The reaction was carried out under a $\mathrm{N}_{2}$ atmosphere at $30{ }^{\circ} \mathrm{C}$. After 90 minutes, a solution of 2(diisopropylamino)ethyl methacrylate (DPA, $12.3 \mathrm{~g}, 57.6 \mathrm{mmol}$ ) in ethanol (15 mL), previously deoxygenated by purging $\mathrm{N}_{2}$ for $1 \mathrm{~h}$ at room temperature, was injected into the flask. After $48 \mathrm{~h}$, the reaction solution was opened to the air, diluted by addition of ethanol $(\approx 200 \mathrm{~mL})$ and left stirring for $1 \mathrm{~h}$. The solution was then passed through a silica column to remove the copper catalyst. After this step, the filtrate was concentrated by rotary evaporation and dialyzed using a $1 \mathrm{kDa}$ MWCO dialysis membrane (Spectrum Labs, Netherland) against chloroform/methanol 2:1 (v/v) $(2 \times 500$ $\mathrm{mL})$, methanol $(2 \times 500 \mathrm{~mL})$, and double-distilled water $(4 \times 2 \mathrm{~L})$. At least $8 \mathrm{~h}$ passed between changes. After dialysis the copolymer was isolated by freeze-drying and characterized by $1 \mathrm{H}-\mathrm{NMR}$ spectroscopy performed on an Avance III 600 spectrometer from Bruker (Billerica, USA), and gel permeation chromatography performed on a GPCMax equipped with an RI detector from Malvern Technologies (Greater Malvern, UK) with acidic water (0.25 vol\% TFA in water) as solvent on a Novamax column (including guard column) from PSS Polymers (Mainz, Germany).

Polymersomes production and characterization. PMPC-PDPA self-assembly of polymersomes, as well as drugs encapsulation, was carried out using the thin film rehydration method. In particular, the polymers was first dissolved in a chloroform:methanol solution (2:1), containing also the 
antibiotics (rifampicin, isoniazid, gentamicin, and lysostaphin) at $1 \mathrm{mg} / \mathrm{mL}$ each. For the production of rhodamine-labeled polymersomes, Rhodamine 6B octadecylester (Sigma) was used (with a 5\% molar ratio with the polymer). The solvent was then evaporated, and the film was rehydrated with endotoxin/LPS-free Dulbecco's PBS (Sigma) for a period of 4 weeks under vigorous stirring, in order to have a final polymer concentration of $10 \mathrm{mg} / \mathrm{mL}$. After this period, the formed polymersomes were purified from the formed tubular structures and only spherical nanoparticles were isolated, according to sucrose-based density gradient centrifugation. ${ }^{32}$ This pre-purified samples were then further purified by size exclusion chromatography for isolating the antibioticsencapsulated nano-vesicles and removing the free drugs. This purified solution was then analyzed by TEM, performed using a FEI Tecnai G2 Spirit electron microscope and/or a JEOL 2100 operating at $200 \mathrm{kV}$ equipped with a CCD camera Orius SC2001 from Gatan. Copper grids were glow discharged and the sample was adsorbed onto the grid. The sample was then stained with $0.75 \mathrm{wt} \%$ phosphotungstic acid (PTA) adjusted to $\mathrm{pH} 7.4$ with $\mathrm{NaOH}$. All the TEM analyses were carried out with dried samples. DLS analyses (for characterizing the nanoparticles size distribution) were carried out using a Zetasizer Nano ZS (Malvern Ltd.) at a copolymer concentration of 0.25 $\mathrm{mg} / \mathrm{mL}$. DLS measurements were based on 12-14 runs, 10-second sub-runs. Samples were analyzed at $25^{\circ} \mathrm{C}$ with a scattering angle of $173^{\circ}$ and a $633 \mathrm{~nm}$ HeNe laser based on a material refractive index (RI) of 1.59 , a dispersant refractive index of 1.330 and a viscosity of 0.89 . Drugs encapsulation was measured by reverse-phase - HPLC measurements. This was performed with Dionex Ultimate 3000 instrument equipped with Variable Wavelength Detector (VWD) to analyze the UV absorption of the polymers at $220 \mathrm{~nm}$ and the enzymes signal at $280 \mathrm{~nm}$. A gradient of $\mathrm{H} 2 \mathrm{O}+$ Tryfluoroacetic acid $0.05 \%$ (TFA) (A) and $\mathrm{MeOH}+\mathrm{TFA} 0.05 \%$ (B) from $0 \mathrm{~min}(5 \% \mathrm{~B})$ to $30 \mathrm{~min}(100 \% \mathrm{~B})$ was used to run the samples trough a $\mathrm{C} 18$ column (Phenomenex). The peak area was integrated by using Chomeleon version 6.8.

Cell culture, in vitro uptake, $\mathrm{NF- \kappa B}$ signaling and trafficking studies. Human monocytes (THP1 cell lines) were differentiated to macrophages through incubation with $5 \mathrm{ng} / \mathrm{mL}$ of phorbol 12myristate 13-acetate (PMA, Sigma) for 48 hours on 24/96 well plates for cell viability/uptake quantification respectively, and on $\mu$-Slide 8 well glass-bottom dishes (ibidi) for confocal analyses. We chose this PMA concentration as it has been found to not undesirable regulate genes expression. ${ }^{15}$ For cell viability, the Thiazolyl Blue Tetrazolium Blue (MTT, Sigma) method was used. Briefly, cells were seeded at a concentration of $5 \cdot 10^{3}$ cells/well in a 96 well plate overnight (O.N.). Increasing concentrations of polymersomes were then added in the growth media, namely $0.1,0.5$, and $1 \mathrm{mg} / \mathrm{mL}$, for periods of 24,48 , and 72 hours. The medium growth was then removed, and an acidified solution of isopropanol was added to dissolve the water insoluble MTT formazan. The solubilized blue crystals were measured colorimetrically at $570 \mathrm{~nm}$ (plate reader ELx800, BioTek). Viability assays were also carried out incubating cells with $10 \mu \mathrm{M}$ Acetoxymethyl (AM) Calcein staining (Invitrogen) for 1 hour, followed by confocal microscopy analyses (Leica TCS SP8). For uptake quantification, THP-1 cells were incubated with rhodamine-labeled polymersomes $(0.1 \mathrm{mg} / \mathrm{mL})$ for 8,24 , and 72 hours, followed by 3 steps of PBS washing and SDSbased cell lysis. Cell debris were then removed by centrifugation, and the rhodamine-polymers present in the supernatant quantified by HPLC.

NF- $\kappa \mathrm{B}$ signaling imaging was preformed using CLSM. Firstly, THP-1 cells were seeded at a concentration of $5 \times 10^{3}$ cells per $\mu$-Slide 8 well glass-bottom dishes (ibidi) and differentiated as above mentioned. Differentiated macrophages were either untreated (CTRL) or incubated with PMPC-PDPA polymersomes ( $1 \mathrm{mg} / \mathrm{mL})$, and LPS (10 $\mathrm{ng} / \mathrm{mL}$, CTRL + ) for $24 \mathrm{~h}$ in a humidified atmosphere, $95 \%$ air, $5 \% \mathrm{CO}_{2}$ at $37{ }^{\circ} \mathrm{C}$. Following treatment, cells were washed with DPBS and 
fixed using 3.7\% formaldehyde (Sigma-Aldrich, Dorset, UK) for $10 \mathrm{~min}$ at room temperature (RT). After fixation, cells were washed with DPBS and permeabilized with $0.2 \%$ Triton-X (SigmaAldrich, Dorset, UK) for a further $10 \mathrm{~min}$ at RT. Then, the immunostaining blocking was performed using 5\% bovine serum albumin (BSA) (Sigma-Aldrich, Dorset, UK), to prevent unspecific antibody binding. After $1 \mathrm{~h}$ at RT, cells were incubated with NF-kB p65 Antibody(F-6) Alexa Fluor ${ }^{\circledR} 647$ (Santa Cruz Biotechnology Inc., Heidelberg, Germany) diluted in 1\% BSA overnight in a humidified chamber at $4{ }^{\circ} \mathrm{C}$. The following day, cells were washed with DPBS and the nucleus was stained with Hoescht 33342 (Sigma-Aldrich, Dorset, UK) for $10 \mathrm{~min}$ at RT before CLSM imaging. At least 10 different regions of the petri dishes were acquired and the NF- $\mathrm{BB}$ nuclear translocation analysis was evaluated by co-localization quantification (Pearson's correlation coefficient) of the NF- $\mathrm{BB}$ and nucleus fluorescence intensity signals using Fiji ImageJ software (version 2.0).

For assessing the endocytic pathways of polymersomes, THP-1 cells have been incubated with Cy5-PMPC-PDPA polymersomes for 30 minutes. After 3 washing steps with PBS, cells have been fixed with formaldehyde (3.7\%) for 10 minutes, washed again with PBS 3 times, blocked with 5\% BSA for 1 hour at room temperature and incubated with anti-EEA1 antibody (PA1-0639 EEA1 Invitrogen) in $1 \%$ BSA overnight at $4{ }^{\circ} \mathrm{C}$. Cells were then washed 3 times in PBS/Tween20 $(0.02 \%)$ and incubated with fluorescently labeled secondary antibody for 1 hour at room temperature before confocal imaging. For analyzing the co-localization with acidic compartments, cells have been inoculated with polymersomes for 30 minutes, washed 3 times with PBS and inoculated with LysoTracker ${ }^{\mathrm{TM}}$ Green DND-26 (ThermoFisher) according to the manufacturer instruction.

\section{In vivo uptake in zebrafish macrophages.}

- For images in Fig 3. We used either zebrafish larvae with fluorescent macrophages $\operatorname{Tg}($ mpeg 1:mcherry) of fluorescent vasculature $\mathrm{Tg}$ (flila:EGFP). General maintenance of zebrafish and performance of injection was performed as described in (Fenaroli et al 2018). For blood infection experiments: at day 2 post fertilization we injected in the posterior cardinal vein $200 \mathrm{CFU}$ of GFP expressing M. marinum. One day later 5 nanoliters of Cy5 labelled PMPC-PDPA polymersomes were injected via the posterior cardinal vein. The imaging was performed using an Andor Dragonfly spinning disc confocal microscope, using a 10X Plan Apo lens (0.45 NA) for imaging the whole embryos (images were stitched together) or a 20X plan Apo len $0.75 \mathrm{NA}$ for imaging the caudal region. For macrophage uptake score a confocal stack of the tail region of zebrafish embryos having red fluorescent macrophages has been acquired at different time points after injection of Cy5 labelled polymersomes. The maximum intensity signal of the nanoparticles and macrophages were thresholder and overlapped; the fluorescence intensity of polymersomes within macrophages was then scored using the program ImageJ. Normalization of the obtained intensity was performed dividing each obtained value by the overall polymersomes fluorescence in the zebrafish embryo. This was done by acquiring an image of the whole zebrafish using a Leica DFC365FX stereomicroscope with a 1.0 $\times$ Plan Apo lens at the magnification of $30 \times$. The scoring of the overall fluorescence in the embryo was performed using the program Fiji. The normalized values were then multiplied by 1000 . The macrophage uptake score formula used for quantification is:

Macrophage uptake score at each time point $(5 \mathrm{~min}, 1 \mathrm{~h}, 8 \mathrm{~h}, 24 \mathrm{~h})=$

\section{$\frac{\text { Polymerosomesfluorescenceinmacrophagesinthetailregion }}{\text { Totalpolymerosomefluorescence }} X 1000$}

For neural tube infection experiments: at day 3 post fertilization we injected, in the neural tube of zebrafish embryos with fluorescent vasculature, $200 \mathrm{CFU}$ of DsRed expressing M. marinum. Four days later we injected 5 nanoliters of Cy5 labelled polymersomes. After 8 hours, images of the whole zebrafish were taken with an Andor Drangonfly Spinning disc confocal using a 10X Plan Apo lens 
(0.45 NA); the images were stitched together. For imaging the granuloma areas, a 20X Plan Apo lens (0.75 NA) was used.

For quantification of accumulation of polymersomes in the granuloma area: images of neural tube infected zebrafish embryos were taken at different times after the intravenous injection of $5 \mathrm{~nL}$ of Cy5 labelled polymersomes. For this analysis we used a Leica DFC365FX stereomicroscope with a $1.0 \times$ Plan Apo lens at the magnification of $30 \times$. Using the program ImageJ, the relative fluorescence of the polymersomes in the granuloma region or in an uninfected region were scored using a rectangle tool of the same size. The value of the uninfected region was considered the background and subtracted from the value in granulomas. This resulting value was normalized by the overall fluorescence of polymersomes in the zebrafish embryo. The final value was multiplied by 100 . The formula is then as follows:

Accumulation in granulomas at each time point $(5 \mathrm{~min}, 1 \mathrm{~h}, 8 \mathrm{~h}, 24 \mathrm{~h})=$

\section{$\frac{\text { Fluorescenceingranuloma-FluorescenceinUninfectedarea }}{\text { Totalzebrafishfluorescence }}$ X 100}

For Spinning disc confocal microscopy: Anesthetized zebrafish larvae were put on a glass-bottom dish (MatTek) and embedded with a solution of low melting point agarose (Sigma). After solidification of the solution, embryo water supplemented with tricaine was added to the dish.

For Leica Stereomicroscope imaging: Anesthetized zebrafish larvae were imaged on a dish having a layer of hardened Agarose 2\% in water.

Videos: The videos were made using the program IMARIS; for Supporting video 1 iso surfaces were employed to facilitate the view of polymersomes within macrophages.

- For supplementary images. Adult zebrafish were maintained according to standard procedures. All experiments were performed on embryos 5 days post fertilization (d.p.f.) or under. Transgenic strains used were the $\operatorname{Tg}\left(\mathrm{mpx}\right.$ :GFP)i114, ${ }^{43}$ and the $\mathrm{Tg}(\mathrm{fms}: \mathrm{GFP}) \operatorname{sh} 377 .{ }^{56} \mathrm{In}$ zebrafish S. aureus imaging experiments, 2 d.p.f LWT zebrafish embryos were injected with 1200 CFUs of CFP-labelled $S$. aureus followed by an injection of $10 \mathrm{mg} / \mathrm{mL}$ rhodamine labelled polymersomes 1 hour later (10\% Rhodamine- PMPC-PDPA, 90\% PMPC-PDPA). Zebrafish were incubated for 2 hours at $28{ }^{\circ} \mathrm{C}$ before analysis by fluorescence microscopy using a pSMT3-mCrimson vector or pSMT3-mCherry vector. ${ }^{46}$ Tuberculous granuloma formation is enhanced by a mycobacterium virulence determinant. Liquid cultures were prepared from bacterial plates with $50 \mu \mathrm{g} / \mathrm{mL}$ hygromycin as previously described. ${ }^{46}$ Specificity of the zebrafish host transcriptome response to acute and chronic mycobacterial infection and the role of innate and adaptive immune components. ${ }^{57}$ Injection inoculum was prepared from overnight liquid cultures with an OD600 of 1, after washing in PBS/Tween 80, and resuspending in 2\% polyvinylpyrrolidone40 (PVP40)/PBS. Injection of $M$. marinum into zebrafish embryos was performed into the blood forming region of the caudal vein at 28-30 h.p.if. Here $200 \mathrm{CFU}$, in a volume of $1 \mathrm{~nL}$, were injected. 1 d.p.i., $3 \mathrm{~nL}$ of Rhodamine labeled polymersomes was injected into the circulation via the Duct of Cuvier. Once injected, the embryos were mounted in 1\% low melting point agarose and fluorescent confocal images and time lapses were generated using a Leica TCS SPE-II microscope using a 40x objective (water immersion, HCX PL APO, 1.10NA).

In vitro and in vivo quantification of bacterial burden. THP-1 cells were differentiated to macrophages in a 96-well plate as previously described. Then we carried out infection with BCG, and M. tuberculosis with a Multiplicity of Infection (M.O.I.) of 10:1 for 24 hours, using antibiotics free RPMI medium. Cells were then washed 3 times in PBS to remove the excess of microorganisms, and incubated with RPMI medium (CTRL), empty polymersomes (CTRL -), 
polymersomes encapsulated with Rifampicin, Isoniazid, or combination of both, and free Rifampicin, Isoniazid, or combination of both free drugs (the antibiotics were all at the same final concentration of $1 \mu \mathrm{g} / \mathrm{mL}$ ). We tested all these formulations for 24 and 72 hours, then macrophages were lysed with $0.05 \%$ SDS, and the CFUs quantified with the SPOTi assay. We carried out serial dilutions of each lysis solution, and $10 \mu \mathrm{L}$ of them were aliquoted on Middelbrock 7H11 agar medium for a period of 25-30 days, or until some colonies were visible. Colony counting was carried out manually. For THP-1 S. aureus experiments, mid-log S. aureus (Newman strain), were centrifuged at 10,000G for 1 minute and resuspended in $1 \mathrm{~mL}$ PBS. 106 CFUs were added to each well (MOI of 5:1). The cells were then placed on ice for 1 hour followed by a further 5 hours in a $37^{\circ} \mathrm{C}$ incubator (total 6 hours incubation). After incubation, gentamicin was added to the media $(150 \mu \mathrm{g} / \mathrm{mL})$ and the cells were left for 30 minutes in an incubator to kill the extracellular bacteria. The samples were removed from the incubator, washed twice with PBS and then replaced with RPMI media containing $15 \mu \mathrm{g} / \mathrm{mL}$ of gentamicin and the treatment or control was added. At each specified time point ( 6.5 hours, 22 hours, and 48 hours post infection) the media was removed, the cells were washed twice with PBS and then $250 \mu \mathrm{L}$ of $1 \%$ Saponin (Sigma Aldrich) was added to lyse the cells. The macrophages were left in the Saponin for 12 minutes in a $37^{\circ} \mathrm{C}$ incubator and then an additional $750 \mu \mathrm{L}$ of PBS was added to the cells and the wells were mixed thoroughly with a pipette. $10 \mu \mathrm{L}$ of the lysed cells were taken and diluted in a 96 well plate with six $1 / 10$ serial dilutions. Three $10 \mu \mathrm{L}$ drops from each dilution were placed onto a labelled blood agar plate, incubated overnight at $37^{\circ} \mathrm{C}$ and the number of viable colonies were counted. For zebrafish in vivo S. aureus experiments, 2 d.p.if. LWT zebrafish were injected with 1200 CFUs of GFP-labelled $S$. aureus. 18 hours after injection zebrafish were viewed under a fluorescent dissecting microscope (Leica MZ10F) and zebrafish with visible abscesses were discarded. 20 hours post infection, zebrafish were injected with $0.5 \mathrm{~nL}$ of $1 \mathrm{mg} / \mathrm{mL}$ polymersomes with $37.5 \mu \mathrm{g} / \mathrm{mL}$ of encapsulated rifampicin or their subsequent controls. Zebrafish embryos were incubated at $28^{\circ} \mathrm{C}$ for a further 20 hours following polymersomes injections and were then homogenized using the PreCellys 24-Dual (Peqlab). The homogenates were serially diluted onto BHI agar plates, placed

in a $37^{\circ} \mathrm{C}$ room and the number of viable colonies was manually counted the following morning. In order to quantify the in vivo microorganism burden, injection of M. marinum into zebrafish embryos was performed into the blood forming region of the caudal vein at 28-30 h.p.f. $100 \mathrm{CFU}$, in a volume of $3 \mathrm{~nL}$, were injected. 1 d.p.i., $1 \mathrm{~nL}$ of PBS (Ctrl), empty polymersomes, free Rifampicin $(3.6 \mathrm{mM})$ or Rifampicin-encapsulated polymersomes $(3.6 \mathrm{mM})$ were injected into the circulation via the Duct of Cuvier. Embryos were imaged at 4 d.p.i. on a wide field Leica DMi8 using a 2.5x objective (air, HC FL PLAN, 0.07NA) with images generated with a Hamamatsu Orca Flash 4.0 V2 camera. Bacterial burden was analyzed suing pixel counting software as previously described. ${ }^{34}$ Data were analyzed using one-way ANOVA (with Bonferroni post-test adjustment) in Prism 6.0 software (GraphPad Software, Sand Diego, CA, USA).

\section{Acknowledgements}

We thank the EPSRC (EP/G062137/1) for the initial in vivo evaluation and ND and GB salary. We are grateful to the ERC for the MEViC ERC-STG project for part of the consumable, GB and LR salary. We sincerely thank the Royal Society Newton International Fellowship and the Marie Skłodowska-Curie Individual Fellowship PHANTOM (795224) for supporting LR research activities and most of TB-related consumables. We thank the MRC doctoral training account for JR studentship, the Wellcome Trust for PME fellowship, the NC3R for AP salary. 


\section{Bibliography}

(1) Ginhoux, F.; Jung, S. Monocytes and Macrophages: Developmental Pathways and Tissue Homeostasis. Nature Reviews Immunology 2014, 14(6), 392-404.

(2) Price, J. V.; Vance, R. E. The Macrophage Paradox. Immunity 2014, 41(5), 685-693.

(3) Portnoy, D. A.; Chen, C.; Mitchell, G. Strategies Used by Bacteria to Grow in Macrophages. Microbiology Spectrum 2016, 4(3), 1-22.

(4) Ray, K.; Marteyn, B.; Sansonetti, P. J.; Tang, C. M. Life on the inside: The Intracellular Lifestyle of Cytosolic Bacteria. Nature Reviews Microbiology 2009, 7(5), 333-340.

(5) Kagan, J. C.; Roy, C. R. Legionella Phagosomes Intercept Vesicular Traffic from Endoplasmic Reticulum Exit Sites. Nature Cell Biology 2002, 4, 945-954.

(6) Sindhwani, A.; Arya, S. B.; Kaur, H.; Jagga, D.; Tuli, A.; Sharma, M. Salmonella Exploits the Host Endolysosomal Tethering Factor HOPS Complex to Promote Its Intravacuolar Replication. PLoS Pathogens 2017, 13(10), e1006700.

(7) Feuerstein, R.; Kolter, J.; Henneke, P. Dynamic Interactions between Dermal Macrophages and Staphylococcus aureus . Journal of Leukocite Biology 2017, 101(1), 99-106.

(8) Horn, J.; Stelzner, K.; Rudel, T.; Fraunholz, M. Inside Job: Staphylococcus aureus Host-Pathogen Interactions. International Journal of Medical Microbiology 2018, 308(6), 607-624.

(9) Ferrari, G.; Langen, H.; Naito, M.; Pieters, J. A Coat Protein on Phagosomes Involved in the Intracellular Survival of Mycobacteria. Cell 1999, 97(4) 435-447.

(10) Kaufmann, S. H. E. How Can Immunology Contribute to the Control of Tuberculosis? Nature Reviews Immunology 2001, 1, 20-30.

(11) Cambier, C. J.; Falkow, S.; Ramakrishnan, L. Host Evasion and Exploitation Schemes of Mycobacterium tuberculosis. Cell 2014, 159(7), 1497-509.

(12) Lange, C.; Alghamdi, W. A.; Al-Shaer, M. H.; Brighenti, S.; Diacon, A. H.; DiNardo, A. R.; Grobbel, H. P.; Gröschel, M. I.; von Groote-Bidlingmaier, F.; Hauptmann, M.; et al. Perspectives for Personalized Therapy for Patients with Multidrug-Resistant Tuberculosis. Journal of Internal Medicine 2018, 284(2), 163-188

(13) O’Donnell, M. R.; Padayatchi, N.; Daftary, A.; Orrell, C.; Dooley, K. E.; Rivet Amico, K.; Friedland, G. Antiretroviral Switching and Bedaquiline Treatment of Drug-Resistant Tuberculosis HIV CoInfection. The Lancet HIV 2019, 6(3), e201-e204.

(14) Greenwood, D. J.; Silva, M.; Santos, D.; Huang, S.; Russell, M. R. G.; Collinson, L. M.; Macrae, J. I.; West, A.; Jiang, H.; Gutierrez, M. G. Subcellular Antibiotic Visualization Reveals a Dynamic Drug Reservoir in Infected Macrophages. Science 2019, 364(6447), 1279-1282.

(15) McKinney, J. D.; Höner Zu Bentrup, K.; Muñoz-Elias, E. J.; Miczak, A.; Chen, B.; Chan, W. T.; Swenson, D.; Sacchettini, J. C.; Jacobs, W. R.; Russell, D. G. Persistence of Mycobacterium tuberculosis in Macrophages and Mice Requires the Glyoxylate Shunt Enzyme Isocitrate Lyase. Nature 2000, 406(6797), 735-738.

(16) Peyron, P.; Vaubourgeix, J.; Poquet, Y.; Levillain, F.; Botanch, C.; Bardou, F.; Daffé, M.; Emile, J. F.; Marchou, B.; Cardona, P. J.; et al. Foamy Macrophages from Tuberculous Patients' Granulomas Constitute a Nutrient-Rich Reservoir for M. tuberculosis Persistence. PLoS Pathogens 2008, 4(11), e1000204.

(17) Discher, B. M.; Won, Y. Y.; Ege, D. S.; Lee, J. C. M.; Bates, F. S.; Discher, D. E.; Hammer, D. A. Polymersomes: Tough Vesicles Made from Diblock Copolymers. Science 1999, 284(5417), 11431146.

(18) Contini, C.; Pearson, R.; Wang, L.; Messager, L.; Gaitzsch, J.; Rizzello, L.; Ruiz-Perez, L.; Battaglia, G. Bottom-Up Evolution of Vesicles from Disks to High-Genus Polymersomes. iScience 2018, 7, 132144.

(19) Messager, L.; Gaitzsch, J.; Chierico, L.; Battaglia, G. Novel Aspects of Encapsulation and Delivery Using Polymersomes. Current Opinion in Pharmacology 2014, 18, 104-111.

(20) Guan, L.; Rizzello, L.; Battaglia, G. Polymersomes and Their Applications in Cancer Delivery and Therapy. Nanomedicine 2015, 10(17), 2757-2780.

(21) Lomas, H.; Canton, I.; MacNeil, S.; Du, J.; Armes, S. P.; Ryan, A. J.; Lewis, A. L.; Battaglia, G. Biomimetic PH Sensitive Polymersomes for Efficient DNA Encapsulation and Delivery. Advanced Material 2007, 19(23), 4238-4243.

(22) Wang, L.; Chierico, L.; Little, D.; Patikarnmonthon, N.; Yang, Z.; Azzouz, M.; Madsen, J.; Armes, S. 
P.; Battaglia, G. Encapsulation of Biomacromolecules within Polymersomes by Electroporation. Angewandte Chemie International Edition 2012, 51(44), 11122-11125.

(23) Colley, H. E.; Hearnden, V.; Avila-Olias, M.; Cecchin, D.; Canton, I.; Madsen, J.; Macneil, S.; Warren, N.; Hu, K.; McKeating, J. A.; et al. Polymersome-Mediated Delivery of Combination Anticancer Therapy to Head and Neck Cancer Cells: 2D and 3D in Vitro Evaluation. Molecular Pharmaceutics 2014, 11(4), 1176-1188.

(24) Massignani, M.; Lopresti, C.; Blanazs, A.; Madsen, J.; Armes, S. P.; Lewis, A. L.; Battaglia, G. Controlling Cellular Uptake by Surface Chemistry, Size, and Surface Topology at the Nanoscale. Small 2009, 5(21), 2424-2432.

(25) Robertson, J. D.; Ward, J. R.; Avila-Olias, M.; Battaglia, G.; Renshaw, S. A. Targeting Neutrophilic Inflammation Using Polymersome-Mediated Cellular Delivery. The Journal of Immunology 2017, 198(9), 3596-3604.

(26) Yu, H.; Zou, Y.; Wang, Y.; Huang, X.; Huang, G.; Sumer, B. D.; Boothman, D. A.; Gao, J. Overcoming Endosomal Barrier by Amphotericin B-Loaded Dual PH-Responsive PDMA- b-PDPA Micelleplexes for SiRNA Delivery. ACS Nano 2011, 5(11), 9246-9255.

(27) Luo, M.; Wang, H.; Wang, Z.; Cai, H.; Lu, Z.; Li, Y.; Du, M.; Huang, G.; Wang, C.; Chen, X.; et al. A STING-Activating Nanovaccine for Cancer Immunotherapy. Nature Nanotechnology 2017, 12(7), 648654.

(28) Davis, J. M.; Clay, H.; Lewis, J. L.; Ghori, N.; Herbomel, P.; Ramakrishnan, L. Real-Time Visualization of Mycobacterium-Macrophage Interactions Leading to Initiation of Granuloma Formation in Zebrafish Embryos. Immunity 2002, 17(6), 693-702.

(29) Ramakrishnan, L. Revisiting the Role of the Granuloma in Tuberculosis. Nature Reviews Immunology 2012, 12(5), 352-66.

(30) Sarathy, J. P.; Zuccotto, F.; Hsinpin, H.; Sandberg, L.; Via, L. E.; Marriner, G. A.; Masquelin, T.; Wyatt, P.; Ray, P.; Dartois, V. Prediction of Drug Penetration in Tuberculosis Lesions. ACS Infectious Diseases 2016, 2(8), 552-563.

(31) Dartois, V. The Path of Anti-Tuberculosis Drugs: From Blood to Lesions to Mycobacterial Cells. Nat. Rev. Microbiol. 2014, 12(3), 159-167.

(32) Robertson, J. D.; Rizzello, L.; Avila-Olias, M.; Gaitzsch, J.; Contini, C.; Magon, M. S.; Renshaw, S. A.; Battaglia, G. Purification of Nanoparticles by Size and Shape. Scientific Reports 2016. 6(27494), $1-9$.

(33) Ferguson, S. M.; De Camilli, P. Dynamin, a Membrane-Remodelling GTPase. Nature Reviews Molecular Cell Biology 2012, 13(2) 75-88..

(34) Sabharanjak, S.; Sharma, P.; Parton, R. G.; Mayor, S. GPI-Anchored Proteins Are Delivered to Recycling Endosomes via a Distinct Cdc42-Regulated Clathrin-Independent Pinocytic Pathway. Developmental Cell 2002, 2(4), 411-23.

(35) Kalia, M.; Kumari, S.; Chadda, R.; Hill, M. M.; Parton, R. G.; Mayor, S. Arf6-Independent GPIAnchored Protein-Enriched Early Endosomal Compartments Fuse with Sorting Endosomes via a Rab5/Phosphatidylinositol-3'-Kinase-Dependent Machinery. Molecular Biology of the Cell 2006, 17(8), 3689-3704.

(36) Kumari, S.; Mayor, S. ARF1 Is Directly Involved in Dynamin-Independent Endocytosis. Nature Cell Biology 2008, 10(1), 30-41.

(37) Morrow, I. C.; Parton, R. G. Flotillins and the PHB Domain Protein Family: Rafts Worms and Anaesthetics. Traffic 2005, 6(9):725-740.

(38) Mayor, S.; Pagano, R. E. Pathways of Clathrin-Independent Endocytosis. Nature Reviews Molecular Cell Biology 2007, 8(8), 603-612.

(39) Valacchi, G.; Sticozzi, C.; Lim, Y.; Pecorelli, A. Scavenger Receptor Class B Type I: A Multifunctional Receptor. Annals of the New York Academy of Sciences 2011, 1229, E1-7.

(40) Shen, W. J.; Hu, J.; Hu, Z.; Kraemer, F. B.; Azhar, S. Scavenger Receptor Class B Type i (SR-BI): A Versatile Receptor with Multiple Functions and Actions. Metabolism: Clinical and Experimental 2014, 63(7), 875-886.

(41) Saddar, S.; Carriere, V.; Lee, W. R.; Tanigaki, K.; Yuhanna, I. S.; Parathath, S.; Morel, E.; Warrier, M.; Sawyer, J. K.; Gerard, R. D.; et al. Scavenger Receptor Class B Type I Is a Plasma Membrane Cholesterol Sensor. Circulation Research 2013, 112(1), 140-151.

(42) Marshall-Clarke, S.; Downes, J. E.; Haga, I. R.; Bowie, A. G.; Borrow, P.; Pennock, J. L.; Grencis, R. K.; Rothwell, P. Polyinosinic Acid Is a Ligand for Toll-like Receptor 3. Journal of Biological Chemistry 2007, 282, 24759-24766. 
(43) Renshaw, S. A.; Loynes, C. A.; Trushell, D. M. I.; Elworthy, S.; Ingham, P. W.; Whyte, M. K. B. A Transgenic Zebrafish Model of Neutrophilic Inflammation. Blood 2006, 108(13), 3976-3978.

(44) Prajsnar, T. K.; Cunliffe, V. T.; Foster, S. J.; Renshaw, S. A. A Novel Vertebrate Model of Staphylococcus aureus Infection Reveals Phagocyte-Dependent Resistance of Zebrafish to Non-Host Specialized Pathogens. Cellular Microbiology 2008, 10(11), 2312-2325.

(45) Hepburn, L.; Prajsnar, T. K.; Klapholz, C.; Moreno, P.; Loynes, C. A.; Ogryzko, N. V.; Brown, K.; Schiebler, M.; Hegyi, K.; Antrobus, R.; et al. A Spaetzle-like Role for Nerve Growth Factor $\beta$ in Vertebrate Immunity to Staphylococcus Aureus. Science 2014, 346(6209), 641-646.

(46) Elks, P. M.; Brizee, S.; van der Vaart, M.; Walmsley, S. R.; van Eeden, F. J.; Renshaw, S. A.; Meijer, A. H. Hypoxia Inducible Factor Signaling Modulates Susceptibility to Mycobacterial Infection via a Nitric Oxide Dependent Mechanism. PLoS Pathogens 2013, 9(12), e1003789.

(47) Tobin, D. M.; Ramakrishnan, L. Comparative Pathogenesis of Mycobacterium marinum and Mycobacterium tuberculosis. Cellular Microbiology 2008, 10(5), 1027-1039.

(48) Fenaroli, F.; Westmoreland, D.; Benjaminsen, J.; Kolstad, T.; Skjeldal, F. M.; Meijer, A. H.; Van Der Vaart, M.; Ulanova, L.; Roos, N.; Nyström, B.; et al. Nanoparticles as Drug Delivery System against Tuberculosis in Zebrafish Embryos: Direct Visualization and Treatment. ACS Nano 2014, 8(7), 70147026.

(49) Vibe, C. B.; Fenaroli, F.; Pires, D.; Wilson, S. R.; Bogoeva, V.; Kalluru, R.; Speth, M.; Anes, E.; Griffiths, G.; Hildahl, J. Thioridazine in PLGA Nanoparticles Reduces Toxicity and Improves Rifampicin Therapy against Mycobacterial Infection in Zebrafish. Nanotoxicology. 2016, 10(6), 680688.

(50) Fenaroli, F.; Repnik, U.; Xu, Y.; Johann, K.; Van Herck, S.; Dey, P.; Skjeldal, F. M.; Frei, D. M.; Bagherifam, S.; Kocere, A.; et al. Enhanced Permeability and Retention-like Extravasation of Nanoparticles from the Vasculature into Tuberculosis Granulomas in Zebrafish and Mouse Models. ACS Nano 2018, 12(8), 8646-8661.

(51) Oehlers, S. H.; Cronan, M. R.; Scott, N. R.; Thomas, M. I.; Okuda, K. S.; Walton, E. M.; Beerman, R. W.; Crosier, P. S.; Tobin, D. M. Interception of Host Angiogenic Signalling Limits Mycobacterial Growth. Nature 2015, 517, 612-615

(52) Dal, N. J. K.; Kocere, A.; Wohlmann, J.; Van Herck, S.; Bauer, T. A.; Resseguier, J.; Bagherifam, S.; Hyldmo, H.; Barz, M.; De Geest, B. G.; et al. Zebrafish Embryos Allow Prediction of Nanoparticle Circulation Times in Mice and Facilitate Quantification of Nanoparticle-Cell Interactions. Small 2020, 16(5), 1906719.

(53) Lee, J. J.; Lim, J.; Gao, S.; Lawson, C. P.; Odell, M.; Raheem, S.; Woo, J. I.; Kang, S. H.; Kang, S. S.; Jeon, B. Y.; et al. Glutamate Mediated Metabolic Neutralization Mitigates Propionate Toxicity in Intracellular Mycobacterium tuberculosis. Scientific Reports 2018, 8(1), 8506.

(54) Abreu, R.; Essler, L.; Loy, A.; Quinn, F.; Giri, P. Heparin Inhibits Intracellular Mycobacterium tuberculosis Bacterial Replication by Reducing Iron Levels in Human Macrophages. Scientific Reports 2018, 8(1), 7296.

(55) Simeone, R.; Sayes, F.; Song, O.; Gröschel, M. I.; Brodin, P.; Brosch, R.; Majlessi, L. Cytosolic Access of Mycobacterium tuberculosis: Critical Impact of Phagosomal Acidification Control and Demonstration of Occurrence in vivo. PLoS Pathog. 2015, 11(2), e1004650

(56) Dee, C. T.; Nagaraju, R. T.; Athanasiadis, E. I.; Gray, C.; Fernandez del Ama, L.; Johnston, S. A.; Secombes, C. J.; Cvejic, A.; Hurlstone, A. F. L. CD4-Transgenic Zebrafish Reveal Tissue-Resident Th2- and Regulatory T Cell-like Populations and Diverse Mononuclear Phagocytes. J. Immunol. 2016, 197(9), 3520-3530.

(57) van der Sar, A. M.; Spaink, H. P.; Zakrzewska, A.; Bitter, W.; Meijer, A. H. Specificity of the Zebrafish Host Transcriptome Response to Acute and Chronic Mycobacterial Infection and the Role of Innate and Adaptive Immune Components. Molecular Immunology 2009, 46(11-12), 2317-2332. 